\title{
Phase Topology of Two Vortices of Identical Intensities in a Bose - Einstein Condensate
}

\author{
P. E. Ryabov, S. V. Sokolov
}

A completely Liouville integrable Hamiltonian system with two degrees of freedom describing the dynamics of two vortex filaments in a Bose-Einstein condensate enclosed in a cylindrical trap is considered. For the system of two vortices with identical intensities a bifurcation of three Liouville tori into one is detected. Such a bifurcation is found in the integrable case of Goryachev - Chaplygin - Sretensky in rigid body dynamics.

Keywords: vortex dynamics, Bose-Einstein condensate, completely integrable Hamiltonian systems, bifurcation diagram of momentum mapping, bifurcations of Liouville tori

Received December 31, 2018

Accepted February 13, 2019

The work of S. V. Sokolov (Sections 1, 2) was carried out at MIPT under project 5-100 for state support for leading universities of the Russian Federation and also partially support by RFBR grants 16-01-00809, 18-01-00335 and 18-29-10051. The work of P. E. Ryabov (Sections 3, 4) was supported by RFBR grants 16-01-00170 and 17-01-00846 and was carried out within the framework of the state assignment of the Ministry of Education and Science of Russia (1.2404.2017/4.6).

Pavel E. Ryabov

PERyabov@fa.ru

Financial University under the Government of the Russian Federation

Leningradsky prosp. 49, Moscow, 125993 Russia

Institute of Machines Science, Russian Academy of Sciences

Maly Kharitonyevsky per. 4, Moscow, 101990 Russia

Udmurt State University

ul. Universitetskaya 1, Izhevsk, 426034 Russia

Sergei V. Sokolov

sokolovsv72@mail.ru

Moscow Institute of Physics and Technology (State University)

Institutskiy per. 9, Dolgoprudny, Moscow Region, 141701 Russia

Institute of Machines Science, Russian Academy of Sciences

Maly Kharitonyevsky per. 4, Moscow, 101990 Russia 


\section{Introduction}

The mainstream of vortex analytical dynamics is an integrable model of point vortices on a plane. Studies of the dynamics of vortices in a quantum fluid have shown that quantum vortices behave approximately the same as thin vortex filaments in a classical perfect fluid. A special place is occupied by the vortex structures in the Bose - Einstein condensate obtained for ultracold atomic gases [1]. This article will be concerned with a mathematical model of the dynamics of two vortex filaments in a Bose-Einstein condensate enclosed in a harmonic trap [2-4]. This model leads to a completely Liouville integrable Hamiltonian system with two degrees of freedom, and for this reason, topological methods used in such systems can be applied. Topological methods were successfully used for investigation of the stability problem of absolute and relative choreographies [5-9]. These motions in integrable models correspond, as a rule, to the values of the constant first integrals for which the integrals, considered as functions of phase variables, turn out to be dependent in the sense of the linear dependence of the differentials. The main role in the study of such dependence is played by the bifurcation diagram of the momentum map.

This publication is devoted to the integrable perturbation of the model considered in [10-13]. In this paper, the bifurcation diagram is explicitly determined and bifurcations of Liouville tori are investigated. In the case of a vortex pair consisting of vortices of identical intensity, a bifurcation of three tori into one is detected for some values of physical parameters. This bifurcation was previously encountered by M. P. Kharlamov in studying the phase topology of the integrable Chaplygin - Goryachev - Sretensky case in the dynamics of a rigid body [14] and as one of the features in the form of a 2-atom of a singular layer of Liouville foliation by A. T. Fomenko, A. V. Bolsinov and S. V. Matveev [15]. In the work of A. A. Oshemkov and M. A. Tuzhilin [16], devoted to the splitting of saddle singularities, such a bifurcation turned out to be unstable and its perturbed foliations, one of which is realized in the integrable model under consideration, were given.

\section{Model and Definitions}

Here we follow the original works [2-4] in the description of the model. Let us consider $N$ interacting vortices in a Bose-Einstein condensate enclosed in a harmonic trap and let $\left(x_{k}, y_{k}\right)$ be the position of the $k$ th vortex, $r_{k}=\sqrt{x_{k}^{2}+y_{k}^{2}}$. A single vortex $\left(x_{k}, y_{k}\right)$ in a harmonic trap is well known to precess around the center of the trap with the frequency $\omega_{\text {pr }}$ which can be approximated by $\omega_{\mathrm{pr}}=\omega_{\mathrm{pr}}^{0} /\left(1-r_{k}^{2} / R_{\mathrm{T} F}^{2}\right)$, where the frequency at the trap center is $\omega_{\mathrm{pr}}^{0}=\ln \left(A \frac{\mu}{\Omega}\right) / R_{\mathrm{TF}}^{2}, \mu$ is the chemical potential, $R_{\mathrm{TF}}=\sqrt{2 \mu} / \Omega$ is the so-called Thomas-Fermi (TF) radius, $A=2 \sqrt{2} \pi$ is a numerical constant, and $\Omega=\omega_{r} / \omega_{z}\left(\omega_{r}\right.$ and $\omega_{z}$ being the confining radial and axial frequencies of the harmonic trap, respectively). On the other hand, in the absence of a harmonic trap, two interacting vortices will rotate around each other with a frequency of $\omega_{\text {vort }}=B / r_{k j}^{2}$, where $r_{k j}=\sqrt{\left(x_{k}-x_{j}\right)^{2}+\left(y_{k}-y_{j}\right)^{2}}$ is the distance between the vortices and $B$ is a constant factor. If $\left(x_{k}, y_{k}\right)$ is the position of the $k$ th vortex, the corresponding mathematical model of the dynamics of $N$ interacting vortices in a Bose-Einstein condensate (BEC) enclosed in a harmonic trap is described by the following system of differential equations [2-4]:

$$
\begin{aligned}
& \dot{x}_{k}=-\Gamma_{k} \omega_{\mathrm{pr}} y_{k}-\frac{B}{2} \sum_{j \neq k}^{N} \Gamma_{j} \frac{y_{k}-y_{j}}{r_{k j}^{2}}, \\
& \dot{y}_{k}=\Gamma_{k} \omega_{\mathrm{pr}} x_{k}+\frac{B}{2} \sum_{j \neq k}^{N} \Gamma_{j} \frac{x_{k}-x_{j}}{r_{k j}^{2}}
\end{aligned}
$$


where $\Gamma_{k}$ is the charge of the $k$ th vortex, $(k=1, \ldots, N)$ and $N$ is the total number of interacting vortices.

For convenience, following [4], we can further rescale time to the period of the single vortex precessing near the center of the trap $\left(\tau=t \omega_{\mathrm{p} r}^{0}\right)$ and pass to dimensionless variables using the relations

$$
x_{k}=\tilde{x}_{k} R_{\mathrm{T} F}, \quad y_{k}=\tilde{y}_{k} R_{\mathrm{T} F} .
$$

After rescaling (2.2), the equations of motion (2.1) are written as

$$
\begin{aligned}
x_{k}^{\prime} & =-\Gamma_{k} \frac{y_{k}}{1-r_{k}^{2}}-c \sum_{j \neq k}^{N} \Gamma_{j} \frac{y_{k}-y_{j}}{r_{k j}^{2}}, \\
y_{k}^{\prime} & =\Gamma_{k} \frac{x_{k}}{1-r_{k}^{2}}+c \sum_{j \neq k}^{N} \Gamma_{j} \frac{x_{k}-x_{j}}{r_{k j}^{2}},
\end{aligned}
$$

where the dimensionless parameter $c$ is defined by the formula

$$
c=\frac{B}{2 \ln \left(A \frac{\mu}{\Omega}\right)}
$$

and the prime ()$^{\prime}$ in $(2.3)$ stands for $\frac{d}{d \tau}$.

The equations of motion (2.3) can be represented in Hamiltonian form

$$
\Gamma_{k} x_{k}^{\prime}=\frac{\partial H}{\partial y_{k}}, \quad \Gamma_{k} y_{k}^{\prime}=-\frac{\partial H}{\partial x_{k}}, \quad k=1, \ldots, N .
$$

with the Hamiltonian

$$
H=\frac{1}{2} \sum_{k=1}^{N} \Gamma_{k}^{2} \ln \left(1-r_{k}^{2}\right)-\frac{c}{2} \sum_{k=1}^{N} \sum_{j<k}^{N} \Gamma_{k} \Gamma_{j} \ln \left(r_{k j}^{2}\right) .
$$

In what follows, we restrict our study to the case with two vortices $(N=2)$ of identical intensities $\left(\Gamma_{1}=\Gamma_{2}=1\right)$. In this case, the Hamiltonian (2.5) will be written as

$$
H=\frac{1}{2} \ln \left[1-\left(x_{1}^{2}+y_{1}^{2}\right)\right]+\frac{1}{2} \ln \left[1-\left(x_{2}^{2}+y_{2}^{2}\right)\right]-\frac{c}{2} \ln \left[\left(x_{2}-x_{1}\right)^{2}+\left(y_{2}-y_{1}\right)^{2}\right] .
$$

In [10] and [11], parameter $c$ was taken to be equal to one, however, in a series of physical works $[2,3]$ for the parameter $c$ in the case of a vortex pair consisting of vortices with identical intensities $\left(\Gamma_{1}=\Gamma_{2}=1\right)$ other values of $c=1.35 ; c=0.1$ were taken on the basis of experimental data. In this connection, it is of interest to study the phase topology when the parameter $c$ takes any positive values.

The phase space $\mathcal{P}$ is given as a direct product of two open circles of radius 1 , with a set of vortex collisions punctured out

$$
\mathcal{P}=\left\{\left(x_{1}, y_{1}, x_{2}, y_{2}\right): x_{1}^{2}+y_{1}^{2}<1, x_{2}^{2}+y_{2}^{2}<1\right\} \backslash\left\{x_{1}=x_{2}, y_{1}=y_{2}\right\} .
$$

The Poisson structure on the phase space $\mathcal{P}$ is given in the standard form

$$
\left\{x_{i}, y_{j}\right\}=\frac{1}{\Gamma_{i}} \delta_{i j}
$$

where $\delta_{i j}$ is the Kronecker symbol. 
System (2.4) admits one additional first integral of motion - the moment of vorticity $(N=2$, $\left.\Gamma_{1}=\Gamma_{2}=1\right)$

$$
F=\Gamma_{1}\left(x_{1}^{2}+y_{1}^{2}\right)+\Gamma_{2}\left(x_{2}^{2}+y_{2}^{2}\right) .
$$

The function $F$ and the Hamiltonian $H$ form on $\mathcal{P}$ a complete involutive set of integrals of the system (2.4) $\left(N=2, \Gamma_{1}=\Gamma_{2}=1\right)$. According to the Liouville-Arnold theorem, the regular surface of the common level set of first integrals is a disconnected union of two-dimensional tori filled with conditionally periodic trajectories. We define the integral mapping $\mathcal{F}: \mathcal{P} \rightarrow \mathbb{R}^{2}$, setting $(f, h)=\mathcal{F}(\boldsymbol{\zeta})=(F(\boldsymbol{\zeta}), H(\boldsymbol{\zeta}))$. The mapping $\mathcal{F}$ is also called a momentum map. Denote by $\mathcal{C}$ the set of all critical points of the momentum maps, that is, points at which $\operatorname{rank} d \mathcal{F}(x)<2$. The set of critical values $\Sigma=\mathcal{F}(\mathcal{C} \cap \mathcal{P})$ is called a bifurcation diagram.

\section{Bifurcation Diagram}

To find the bifurcation diagram, we use the method of critical subsystems developed by M.P. Kharlamov in integrable problems of rigid body dynamics [17]. As an application, an analysis of the stability of critical trajectories (that is, nondegenerate singularities of rank 1 of the momentum map) is given by determining the type of trajectory (elliptic/hyperbolic) for each curve from the bifurcation set.

In what follows, we confine our attention to identical intensities $\left(\Gamma_{1}=\Gamma_{2}=1\right)$. Let $\mathcal{N}_{1}$ denote the closure of the set of solutions of the system

$$
x_{1}+x_{2}=0 ; \quad y_{1}+y_{2}=0
$$

and let $\mathcal{N}_{2}$ denote the closure of the set of solutions of the system

$$
F_{1}=0, \quad F_{2}=0,
$$

where

$$
\begin{aligned}
& F_{1}=x_{1} y_{2}-y_{1} x_{2}, \\
& F_{2}=\left(x_{1}^{2}+x_{2}^{2}\right)\left(x_{2}^{2}+y_{2}^{2}\right)\left[x_{1}\left(x_{2}^{2}+y_{2}^{2}\right)-c x_{2}\right]+x_{2}\left[(c-2)\left(x_{2}^{2}+y_{2}^{2}\right)^{2} x_{1}^{2}+c x_{2}^{2}\right] .
\end{aligned}
$$

Then the theorem is valid.

Theorem 1. A set $\mathcal{C}$ of critical points of the momentum map $\mathcal{F}$ is exhausted by a set of solutions of the collection of systems (3.1) and (3.2). The sets $\mathcal{N}_{1}$ and $\mathcal{N}_{2}$ are two-dimensional invariant submanifolds of the system (2.4) with the Hamiltonian (2.6).

Proof. To prove the first statement of the theorem, we need to find points of phase space at which the rank of the map is not maximal. By direct calculations it can be verified that the Jacobi matrix of the momentum map has zero minors of the second order at points $\zeta \in \mathcal{P}$ whose coordinates satisfy the equations of the system (3.1) and (3.2), whence $\mathcal{C}=\mathcal{N}_{1} \cup \mathcal{N}_{2}$. The invariance of relations $\mathcal{N}_{1}$ and $\mathcal{N}_{2}$ can be verified by using the following chains of equalities:

$$
\begin{aligned}
& \left(x_{1}+x_{2}\right)^{\prime}=\left\{x_{1}+x_{2}, H\right\}=\sigma_{1}\left(y_{1}+y_{2}\right)=0 ; \\
& \left(y_{1}+y_{2}\right)^{\prime}=\left\{y_{1}+y_{2}, H\right\}=\sigma_{2}\left(x_{1}+x_{2}\right)=0 ; \\
& F_{1}{ }^{\prime}=\left.\left\{F_{1}, H\right\}\right|_{F_{1}=0}=\sigma_{3} F_{2} ; \quad F_{2}{ }^{\prime}=\left.\left\{F_{2}, H\right\}\right|_{F_{1}=0}=\sigma_{4} F_{2},
\end{aligned}
$$

where $\sigma_{k}$ are some functions of the phase variables.

To determine the bifurcation diagram $\Sigma$, it is convenient to go to the polar coordinates

$$
x_{1}=r_{1} \cos \theta_{1}, \quad y_{1}=r_{1} \sin \theta_{1}, \quad x_{2}=r_{2} \cos \theta_{2}, \quad y_{2}=r_{2} \sin \theta_{2} .
$$


Substitution in (3.1) and (3.2) leads to the equation $\sin \left(\theta_{1}-\theta_{2}\right)=0$, i.e., $\theta_{1}-\theta_{2}=0$ and $\theta_{1}-\theta_{2}=\pi$. The first possibility, unlike the dynamics of two vortex intensities of opposite signs [10], is not realized for any positive values of the parameter $c$. For the second case, i.e., when $\theta_{1}=\theta_{2}+\pi$, we obtain

$$
\left\{\begin{array}{l}
\theta_{1}=\theta_{2}+\pi \\
{\left[\begin{array}{l}
r_{1}=r_{2} \\
\left(r_{1}^{2}+r_{2}^{2}\right)\left(r_{1} r_{2}+c\right)-(c-2) r_{1}^{2} r_{2}^{2}-c=0
\end{array}\right.}
\end{array}\right.
$$

The last equation of the system (3.3) can be rewritten as

$$
-r_{1} r_{2}\left(r_{1}+r_{2}\right)^{2}+c\left(1-r_{1}^{2}\right)\left(1-r_{2}^{2}\right)=0
$$

In such a form (3.4) coincides with an equation in [3] on p. 225301-2, derived entirely from other considerations. Our conclusion thus explains that the equation in [3] on p. 225301-2 determines the radii for critical vortex motions.

The corresponding bifurcation diagram $\Sigma$ is defined on the plane $\mathbb{R}^{2}(f, h)$ and consists of two curves $\gamma_{1}$ and $\gamma_{2}$, where

$$
\begin{aligned}
& \gamma_{1}: h=\ln \left(1-\frac{f}{2}\right)-\frac{c}{2} \ln (2 f), \quad 0<f<2 ; \\
& \gamma_{2}:\left\{\begin{array}{l}
h=\frac{1}{2} \ln \left[\frac{s^{2}(s-1)}{c+s-1}\right]-\frac{1}{2} c \ln \left[\frac{c s^{2}}{c+s-1}\right], \quad s \in\left(1 ; \frac{2(1+\sqrt{c})}{2+\sqrt{c}}\right] . \\
f=\frac{c s^{2}-2(s-1)(c+s-1)}{c+s-1},
\end{array}\right.
\end{aligned}
$$

For the values of the physical parameter $c>3$, the curve $\gamma_{2}$ has a cusp at $s=$ $=\frac{[2-c+\sqrt{c(c-2)}](c-1)}{c-2}$, which coincides with the point of tangency, when $c=3$ and $s=\frac{2(1+\sqrt{c})}{2+\sqrt{c}}$.

As an application, we investigate the stability of critical trajectories that lie in the preimage of the bifurcation curves (3.5). In this case, it suffices to determine the type (elliptic/hyperbolic) at any one point $(f, h)$ of the smooth branch curve $\Sigma[18,19]$.

The type of the critical point $x_{0}$ of rank 1 in an integrable system with two degrees of freedom can be calculated in the following way. One must specify the first integral $F$, such that $d F\left(x_{0}\right)=0$ and $d F \neq 0$ in a neighborhood of this point. The $x_{0}$ is a fixed point for the Hamiltonian vector field $\operatorname{sgrad} F$ and it is possible to calculate the linearization of this field at a given point - the operator $A_{F}$ at the point $x_{0}$. This operator will have two zero eigenvalues, the remaining factor of the characteristic polynomial is $\mu^{2}-C_{F}$, where $C_{F}=\frac{1}{2} \operatorname{trace}\left(A_{F}^{2}\right)$. When $C_{F}<0$ we get a point of center type (the corresponding periodic solution is elliptic, it is a stable periodic solution in phase space, the limit of the concentric family of two-dimensional regular tori), and for $C_{F}>0$ we get a point of saddle type (the corresponding periodic solution is of hyperbolic type, there are motions asymptotic to this solution lying on two-dimensional separatrix surfaces). Here we present explicit expressions for $C_{F}$ only for bifurcation curves $\gamma_{1}$ 


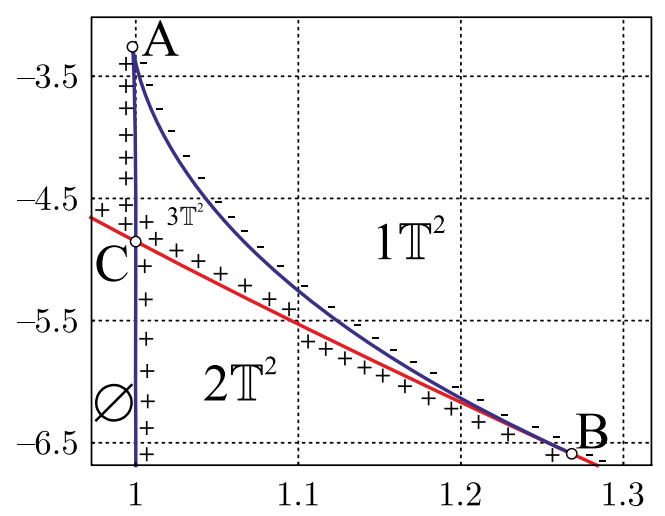

Fig. 1. Enlarged fragment of the bifurcation diagram $\Sigma$.

and $\gamma_{2}$ :

$$
\begin{aligned}
& \gamma_{1}: C_{F}=(4-c) f^{2}+4 c f-4 c, \quad 0<f<2 \\
& \gamma_{2}: C_{F}=(c-2) s^{2}+2(c-1)(c-2) s-2(c-1)^{2}, \quad s \in\left(1 ; \frac{2(1+\sqrt{c})}{2+\sqrt{c}}\right] .
\end{aligned}
$$

Figure 1 shows an enlarged fragment of the bifurcation diagram in the case of identical intensities with $c>3$. The signs "+" and "-" correspond to elliptic (stable) and hyperbolic (unstable) periodic solutions in phase space. As expected, the type change occurs at the cusp $A$ and at the point of tangency $B$ of the bifurcation diagram $\Sigma$.

\section{Bifurcation $3 \mathbb{T}^{2} \rightarrow \mathbb{S}^{1} \times\left(\mathbb{S}^{1} \dot{\cup} \mathbb{S}^{1} \cup \mathbb{S}^{1}\right) \rightarrow \mathbb{T}^{2}$}

Here we confine our attention to identical intensities $\left(\Gamma_{1}=\Gamma_{2}=1\right)$. Perform an explicit reduction to a system with one degree of freedom. For this, in the system $(2.4)(N=2)$ with the Hamiltonian (2.6), we replace the phase variables $\left(x_{k}, y_{k}\right)$ by the new variables $(u, v, \alpha)$ using the formulas:

$$
\begin{aligned}
& x_{1}=u \cos (\alpha)-v \sin (\alpha), \quad y_{1}=u \sin (\alpha)+v \cos (\alpha), \\
& x_{2}=\sqrt{f-u^{2}-v^{2}} \cos (\alpha), \quad y_{2}=\sqrt{f-u^{2}-v^{2}} \sin (\alpha) .
\end{aligned}
$$

The physical variables $(u, v)$ are the Cartesian coordinates of one of the vortices in the coordinate system associated with another vortex rotating around the center of vorticity. The choice of such variables is suggested by the presence of the integral of the moment of vorticity (2.8), which is invariant under the rotation group $S O(2)$. The existence of a one-parameter symmetry group allows one to reduce to a system with one degree of freedom, just as it does in mechanical systems with symmetry [14]. The backward replacement

$$
U=\frac{x_{1} x_{2}+y_{1} y_{2}}{\sqrt{x_{2}^{2}+y_{2}^{2}}}, \quad V=\frac{y_{1} x_{2}-x_{1} y_{2}}{\sqrt{x_{2}^{2}+y_{2}^{2}}}
$$

leads to canonical variables with respect to the bracket $(2.7)\left(\Gamma_{1}=\Gamma_{2}=1\right)$ :

$$
\{U, V\}=-\{V, U\}=1, \quad\{U, U\}=\{V, V\}=0 .
$$

The system with respect to the new variables $(u, v)$ is Hamiltonian

$$
u^{\prime}=\frac{\partial H_{1}}{\partial v}, \quad v^{\prime}=-\frac{\partial H_{1}}{\partial u}
$$


with the Hamiltonian

$$
H_{1}=\frac{1}{2} \ln \left(1-u^{2}-v^{2}\right)+\frac{1}{2} \ln \left(1-f+u^{2}+v^{2}\right)-\frac{1}{2} c \ln \left[f-2 \sqrt{f-u^{2}-v^{2}} u\right] .
$$

The rotation angle $\alpha(t)$ of the rotating coordinate system satisfies the differential equation

$$
\alpha^{\prime}=\frac{R(u, v)}{Q(u, v)}
$$

where

$$
\begin{aligned}
& R(u, v)=2\left\{\sqrt{f-u^{2}-v^{2}}\left[b\left(1-f+u^{2}+v^{2}\right)+f\right]-u\left[(b-2)\left(u^{2}+v^{2}-f\right)+b\right]\right\}, \\
& Q(u, v)=\sqrt{f-u^{2}-v^{2}}\left(1-f+u^{2}+v^{2}\right)\left[f-2 \sqrt{f-u^{2}-v^{2}} u\right] .
\end{aligned}
$$

The fixed points of the reduced system (4.1) are determined by the critical points of the reduced Hamiltonian (4.2) and correspond to the relative equilibria of vortices in the system (2.4). For a fixed value of the integral of the moment of vorticity $f$, the regular levels of the reduced Hamiltonian are compact and motions occur along closed curves. It can be shown that the critical values of the reduced Hamiltonian determine the bifurcation diagram (3.5). For a segment of the bifurcation curve $(A B)$ (Fig. 1), the motion on the plane $(u, v)$ occurs along a curve that is topologically structured as $\mathbb{S}^{1} \dot{\cup} \mathbb{S}^{1} \dot{\cup} \mathbb{S}^{1}$ (Fig. 2b), and the integral critical surface is a trivial bundle over $\mathbb{S}^{1}$ with a layer $\mathbb{S}^{1} \cup \mathbb{S}^{1} \cup \mathbb{S}^{1}$.

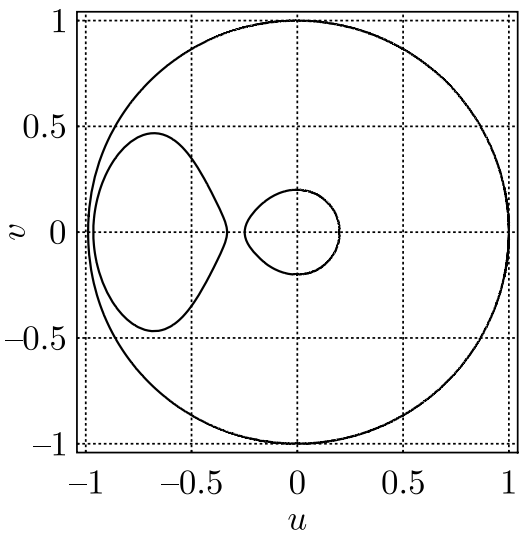

(a)

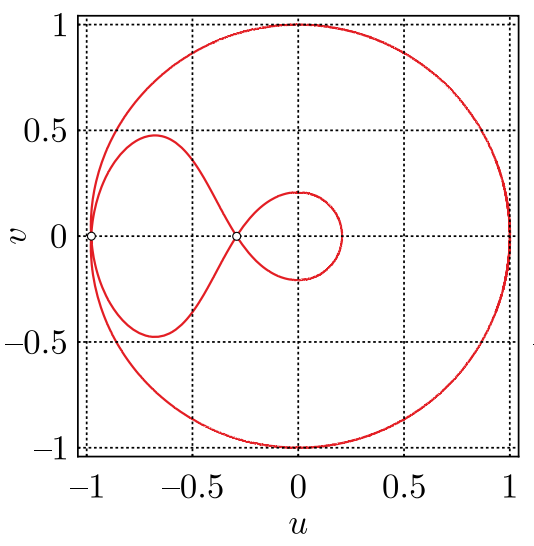

(b)

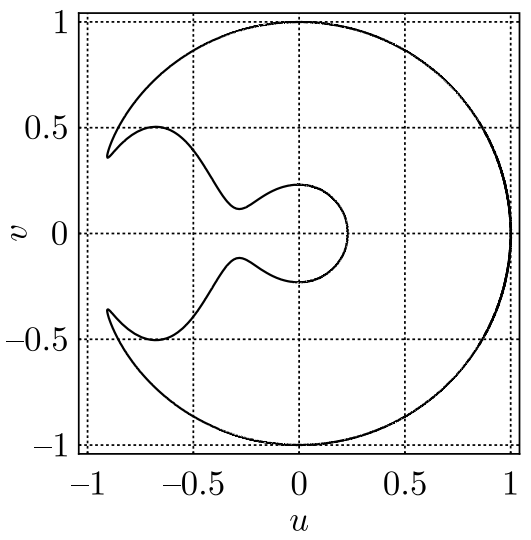

(c)

Fig. 2. Level curves of the reduced Hamiltonian $H_{1}$ for $c>3$.

When passing through the section of the curve $(A B)$ of the bifurcation diagram $\Sigma$ for $c>3$ (Fig. 1), the bifurcation of three tori into one occurs as follows: $3 \mathbb{T}^{2} \rightarrow \mathbb{S}^{1} \times\left(\mathbb{S}^{1} \cup \mathbb{S}^{1} \cup \mathbb{S}^{1}\right) \rightarrow \mathbb{T}^{2}$. With the help of the level curves of the reduced Hamiltonian in Fig. 2, this bifurcation is clearly demonstrated $\left(h_{1}=-4.5\right.$ for a) $f_{1}=1.04$; b) $f_{2}=1.042957$; c) $\left.f_{3}=1.05\right)$.

After splitting the phase space into areas in which the number of tori remains unchanged, explicitly defining the bifurcation diagram and the bifurcations of Liouville tori, we can formulate the problem of classifying the absolute motions of vortices, as well as determining the topological type of three-dimensional isoenergy manifolds.

\section{Acknowledgements}

The authors thank A. V. Borisov for valuable discussions. 


\section{References}

[1] Fetter, A. L., Rotating Trapped Bose-Einstein Condensates, Rev. Mod. Phys., 2009, vol. 81, no. 2, pp. 647-691.

[2] Torres, P. J., Kevrekidis, P. G., Frantzeskakis, D. J., Carretero-González, R., Schmelcher, P., and Hall, D. S., Dynamics of Vortex Dipoles in Confined Bose-Einstein Condensates, Phys. Lett. A, 2011, vol. 375, no. 33, pp. 3044-3050.

[3] Navarro, R., Carretero-González, R., Torres, P. J., Kevrekidis, P. G., Frantzeskakis, D. J., Ray, M. W., Altuntaş, E., and Hall, D. S., Dynamics of a Few Corotating Vortices in Bose-Einstein Condensates, Phys. Rev. Lett., 2013, vol. 110, no. 22, 225301, 6 pp.

[4] Koukouloyannis, V., Voyatzis, G., and Kevrekidis, P. G., Dynamics of Three Noncorotating Vortices in Bose-Einstein Condensates, Phys. Rev. E, 2014, vol. 89, no. 4, 042905, 14 pp.

[5] Borisov, A. V. and Kilin, A. A., Stability of Thomson's Configurations of Vortices on a Sphere, Regul. Chaotic Dyn., 2000, vol. 5, no. 2, pp. 189-200.

[6] Kilin, A.A., Borisov, A. V., and Mamaev, I.S., The Dynamics of Point Vortices inside and outside a Circular Domain, in Basic and Applied Problems of the Theory of Vortices, A. V. Borisov, I. S. Mamaev, M. A. Sokolovskiy (Eds.), Izhevsk: R\&C Dynamics, Institute of Computer Science, 2003, pp. 414-440 (Russian).

[7] Borisov, A. V., Mamaev, I. S., and Kilin, A. A., Absolute and Relative Choreographies in the Problem of Point Vortices Moving on a Plane, Regul. Chaotic Dyn., 2004, vol. 9, no. 2, pp. 101-111.

[8] Borisov, A.V., Kilin, A.A., and Mamaev, I.S., The Dynamics of Vortex Rings: Leapfrogging, Choreographies and the Stability Problem, Regul. Chaotic Dyn., 2013, vol. 18, nos. 1-2, pp. 33-62.

[9] Borisov, A. V., Ryabov, P.E., and Sokolov, S. V., Bifurcation Analysis of a Problem on the Motion of a Cylinder and a Point Vortex in an Ideal Fluid, Math. Notes, 2016, vol. 99, nos. 5-6, pp. 834-839 (https://doi.org/10.1134/S0001434616050217); see also: Mat. Zametki, 2016, vol.99, no. 6 , pp. 848-854.

[10] Sokolov, S. V. and Ryabov, P. E., Bifurcation Analysis of the Dynamics of Two Vortices in a BoseEinstein Condensate. The Case of Intensities of Opposite Signs, Regul. Chaotic Dyn., 2017, vol. 22, no. 8, pp. 979-998.

[11] Sokolov, S. V. and Ryabov, P.E., Bifurcation Diagram of the Two Vortices in a Bose-Einstein Condensate with Intensities of the Same Signs, Dokl. Math., 2018, vol.97, no. 3, pp. 286-290; see also: Dokl. Akad. Nauk, 2018, vol. 480, no. 6, pp.652-656.

[12] Ryabov, P. E., Bifurcation Diagram of One Perturbed Vortex Dynamics Problem, arXiv:1811.10512 (2018).

[13] Ryabov, P. E., On One Unstable Bifurcation in the Dynamics of Vortex Structure, arXiv:1812.03563 (2018).

[14] Kharlamov, M.P., Topological Analysis of Integrable Problems of Rigid Body Dynamics, Leningrad: Leningr. Gos. Univ., 1988 (Russian).

[15] Bolsinov, A. V., Matveev, S. V., and Fomenko, A. T., Topological Classification of Integrable Hamiltonian Systems with Two Degrees of Freedom. List of Systems of Small Complexity, Russian Math. Surveys, 1990, vol.45, no.2, pp.59-94; see also: Uspekhi Mat. Nauk, 1990, vol.45, no. 2(272), pp. 49-77, 240.

[16] Oshemkov, A. A. and Tuzhilin, M. A., Integrable Perturbations of Saddle Singularities of Rank 0 of Integrable Hamiltonian Systems, Sb. Math., 2018, vol.209, no.9, pp. 1351-1375; see also: Mat. Sb., 2018, vol. 209, no. 9, pp. 102-127.

[17] Kharlamov, M.P., Extensions of the Appelrot Classes for the Generalized Gyrostat in a Double Force Field, Regul. Chaotic Dyn., 2014, vol. 19, no. 2, pp. 226-244.

[18] Bolsinov, A. V., Borisov, A. V., and Mamaev, I. S., Topology and Stability of Integrable Systems, Russian Math. Surveys, 2010, vol.65, no. 2, pp. 259-318; see also: Uspekhi Mat. Nauk, 2010, vol. 65, no. 2, pp. 71-132.

[19] Ivanov, A.P., On Singular Points of Equations of Mechanics, Dokl. Math., 2018, vol.97, no.2, pp. 167-169; see also: Dokl. Akad. Nauk, 2018, vol.479, no. 5, pp. 493-496. 